\title{
Ocorrência de lesão por pressão em pacientes internados em um hospital-escola
}

\author{
Occurrence of pressure injury in patients hospitalized in a school hospital
}

Aparición de lesión por presión en pacientes internados en un hospital-escuela

Juliana Magali Barbosa', Geraldo Magela Salomé

ORCID IDs

Barbosa JM ID https://orcid.org/0000-0003-0583-8438

Salomé GM (D) https://orcid.org/0000-0002-7315-4866

\section{COMO CITAR}

Barbosa JM; Salomé GM. Ocorrência de lesão por pressão em pacientes internados em um hospital-escola. ESTIMA, Braz. J. Enterostomal Ther., 16: e2718. https://doi.org/10.30886/estima.v16.523_PT.

A autora Barboa JM agradece a bolsa de estudo do Programa de Bolsa de Iniciação Científica (PROBIC) da Fundação de Amparo à Pesquisa do Estado de Minas Gerais (FAPEMIG)

\section{RESUMO}

Objetivo: Avaliar a ocorrência e fatores de risco para o desenvolvimento de lesão por pressão (LP) em pacientes internados nas clínicas médica, cirúrgica e de observação do pronto-socorro de um hospital universitário. Métodos: Estudo transversal, descritivoexploratório, de caráter epidemiológico. Os pacientes foram avaliados por meio de exame físico, três vezes por semana, durante dois meses consecutivos, entre junho e novembro de 2016. Resultados: A frequência de LP foi de 29\% ( $n=9)$ na clínica médica, $16 \%$ ( $n=4)$ na clínica cirúrgica e 53,8\% ( $n=7)$ na observação do pronto-socorro. Segundo a escala de Braden, sete $(30,4 \%)$ pacientes da clínica médica apresentaram risco elevado e dois (25\%) risco moderado; três (27,3\%) pacientes da clínica cirúrgica apresentaram risco elevado e um $(7,1 \%)$ risco moderado; e sete $(58,3 \%)$ pacientes na observação do pronto-socorro apresentaram risco elevado. Os fatores de risco associados aos participantes que desenvolveram LP foram: restrição no leito, o uso de cateteres ou dispositivos, droga vasoativa, fralda, ventilação mecânica, sedativos, inconsciência, jejum alimentar e tempo de internação acima de 10 dias. Conclusão: Houve alta frequência de LP nas clínicas médica e cirúrgica e na observação do pronto-socorro. A maioria dos pacientes apresentou risco elevado para desenvolver LP.

DESCRITORES: Lesão por pressão; Medição de risco; Cuidados de enfermagem; Estomaterapia.

\footnotetext{
1 Universidade do Vale do Sapucaí, Faculdade de Ciências Médicas Dr. José Antônio Garcia Coutinho, Escola de Enfermagem - Pouso Alegre/MG - Brasil.

Autor correspondente: Geraldo Magela Salomé | Universidade do Vale do Sapucaí - Campus Fátima | Av. Prefeito Tuany Toledo, 470 | CEP: 37550-000 - Pouso Alegre/MG - Brasil | E-mail: salomereiki@univas.edu.br

Recebido: Ago 282017 | Aceito: Out 172017
} 


\begin{abstract}
Objective: to evaluate the occurrence and risk factors for the development of pressure injury (PI) in patients admitted in medical and surgical clinics and in observation at the emergency room of a university hospital. Methods: Cross-sectional, descriptive-exploratory, epidemiological study. Patients were assessed by physical examination three times a week for two consecutive months between June and November 2016. Results: the frequency of PI was 29\% $(n=9)$ in the medical clinic, $16 \%(n=4)$ in the surgical clinic and $53.8 \%$ $(n=7)$ in observation at the emergency room. According to the Braden scale, seven (30.4\%) patients in the medical clinic presented high risk and two (25\%) moderate risk; three (27.3\%) patients from the surgical clinic presented a high risk and one (7.1\%) moderate risk; and seven (58.3\%) patients in observation at the emergency room were high risk. The risk factors associated with the participants who developed PI were: restriction in the bed, use of catheters or devices, vasoactive drug, diaper, mechanical ventilation, sedatives, unconsciousness, food fasting and hospitalization time over 10 days. Conclusion: there was a high frequency of PI in the medical and surgical clinics and in observation at the emergency room. Most patients were high risk for developing PI.
\end{abstract}

DESCRIPTORS: Pressure injury; Risk measurement; Nursing care; Stomatherapy.

\title{
RESUMEN
}

Objetivo: Evaluar la incidencia y factores de riesgo para el desarrollo de lesión por presión (LP) en pacientes internados en las clínicas médica, quirúrgica y de observación de la sala de urgencia, de un hospital universitario. Métodos: Estudio transversal, descriptivoexploratorio, de carácter epidemiológico. Los pacientes fueron evaluados por medio de examen físico, tres veces por semana, durante dos meses consecutivos, entre junio y noviembre de 2016. Resultados: La frecuencia de LP fue de $29 \%$ ( $n=9$ ) en la clínica médica, 16\% $(n=4)$ en la clínica quirúrgica y 53,8 \% $(n=7)$ en la observación de la sala de urgencia. Según la escala de Braden, siete (30,4\%) pacientes de la clínica médica presentaron riesgo elevado y dos (25\%) riesgo moderado; tres (27,3\%) pacientes de la clínica quirúrgica presentaron riesgo elevado y uno $(7,1 \%)$ riesgo moderado; y siete $(58,3 \%)$ pacientes en la observación de la sala de urgencia presentaron riesgo elevado. Los factores de riesgo asociados a los participantes que desarrollaron LP fueron: restricción en la cama, el uso de catéteres o dispositivos, droga vasoactiva, pañal, ventilación mecánica, sedantes, inconsciencia, ayuno alimenticio y tiempo de internación por encima de 10 días. Conclusión: Hubo alta frecuencia de LP en las clínicas médica y quirúrgica y en la observación de la sala de urgencia. La mayoría de los pacientes presentó riesgo elevado para desarrollar LP.

DESCRIPTORES: Lesión por presión; Medición de riesgo; Cuidados de enfermería; Estomaterapia.

\section{INTRODUÇÃO}

As lesões por pressão (LP) são lesões localizadas sobre uma proeminência óssea e em partes moles, podendo ser superficiais ou profundas, de etiologia isquêmica, secundárias a um aumento de pressão externa ${ }^{1,2}$. Estudos nacionais e internacionais apresentam taxas de incidência de LP que variam de $1 \mathrm{a} 35 \%$ em pacientes hospitalizados ${ }^{3-7}$. A prevalência de LP em hospital geral é bastante variável na literatura nacional e internacional, oscilando de 7 a $29 \%$ e de 15 a 25\% em pacientes com doenças crônicas ${ }^{4,8,9}$.

Alguns dos pacientes hospitalizados apresentam alto risco para desenvolver LP devido ao comprometimento neurológico e a limitações de mobilidade. Muitos estão recebendo drogas sedativas e analgésicas, as quais diminuem a percepção sensorial e prejudicam a mobilidade; outros estão desnutridos ou obesos. Outros fatores, tanto intrínsecos como extrínsecos, incluindo idade, uso de drogas vasoativas, estado nutricional, anemias, infecções, sensibilidade cutânea, incontinências, instabilidade hemodinâmica, agitação, umidade, fricção e cisalhamento, também contribuem para o desenvolvimento de LP. Pacientes com essas características representam um grupo prioritário para o profissional de saúde, pois apresentam fatores de risco para o desenvolvimento de LP. Tendo isso em vista, o profissional de saúde deve utilizar uma escala de avaliação dos fatores de risco. Ao identificar os fatores de risco, o profissional deverá adotar medidas que diminuam a pressão, a fricção e o cisalhamento na pele sobre proeminências ósseas, como uso de colchões adequados, coxim, mudanças de decúbito, coberturas com placa de hidrocoloide, filme transparente de poliuretano, entre outras ${ }^{3,10-12}$.

Para que o profissional possa desenvolver programas de medidas preventivas para diminuição da ocorrência de LP, é necessário que ele tenha conhecimento epidemiológico sobre sua prevalência e incidência, dos fatores que mais contribuem para seu desenvolvimento e dos que controlam sua presença ou ausência. Os indicadores epidemiológicos têm grande utilidade, pois medem a evolução temporal de um problema. Prevalência e incidência são os indicadores mais 
utilizados nos estudos das LP. Cada vez mais, enfermeiros, profissionais de saúde e gestores têm a responsabilidade de garantir a qualidade da assistência prestada aos pacientes e adequar essa assistência de acordo com os resultados obtidos $^{13-15}$.

\section{OBJETIVO}

Avaliar a ocorrência e os fatores de risco para o desenvolvimento de LP em pacientes na clínica médica, cirúrgica e unidade de observação do pronto-socorro de um hospital universitário do sul do estado de Minas Gerais (MG).

\section{MÉTODOS}

Esse é um estudo transversal de caráter epidemiológico.

$\mathrm{O}$ projeto de pesquisa foi aprovado pelo Comitê de Ética e Pesquisa da Faculdade de Ciências Médicas Dr. José Antônio Garcia Coutinho da Universidade do Vale do Sapucaí (Univás), sob o parecer Certificado de Apresentação para Apreciação Ética (CAAE) 01883312.6.0000.5102. Todos os participantes ou acompanhantes assinaram o Termo de Consentimento Livre e Esclarecido.

A população-alvo foi composta por 69 pacientes com mais de 18 anos, de ambos os sexos, internados sem LP no Hospital das Clínicas Samuel Libânio da Univás, localizado em Pouso Alegre, MG, e que permaneceram internados nos setores de clínica médica, clínica cirúrgica e observação do pronto-socorro por período igual ou superior a 48 horas.

Os pacientes com idade igual ou inferior a 18 anos e pacientes que permaneceram internados nos setores hospitalares por período inferior a 48 horas não foram incluídos no estudo. Foram excluídos os pacientes que, no decorrer da coleta dos dados, recusaram-se a ser avaliados, ou seja, não permitiram a inspeção da pele pelo pesquisador durante o estudo.

A coleta de dados foi realizada nas segundas, quartas e sextas-feiras, durante dois meses consecutivos, em cada setor. A coleta de dados foi iniciada em junho 2016 e terminou em novembro de 2016. Os instrumentos utilizados para a coleta de dados foram: um questionário para registro de dados sociodemográficos (faixa etária, gênero, cor, tempo de internação), dados clínicos (diabetes melito, hipertensão arterial, tabagismo), dados relacionados aos fatores de risco (índice de massa corporal, restrição no leito, uso de cateteres e dispositivos, uso de fralda, incontinência urinária, ventilação mecânica, uso de sedação, uso de droga, agitação motora, jejum alimentar) e escala de Braden.

Optou-se por utilizar a escala de Braden por ter sido traduzida e validada para a língua portuguesa. Essa escala é composta por seis subescalas que medem o grau de percepção sensorial, umidade, atividade física, nutrição, mobilidade, fricção e cisalhamento. Todas as subescalas são graduadas de 1 a 4, exceto fricção e cisalhamento, cuja variação é de 1 a 3. A pontuação da escala varia de 6 a 23 pontos, sendo: risco muito alto, de 6 a 9 pontos; risco alto de 10 a 12 pontos; risco moderado, de 13 a 14 pontos, risco baixo, de 15 a 18 pontos; e sem risco, acima de 19 pontos. No presente estudo foram considerados pacientes em risco elevado de LP aqueles que apresentavam risco alto e risco muito alto de desenvolvimento de LP, ou seja, com escores entre 6 a 12 pontos $^{16}$.

Para análise estatística, foram utilizados o teste exato de Fisher e o teste qui-quadrado. Considerou-se como nível de significância estatística para os testes o valor de $\mathrm{p}<0,05$.

\section{RESULTADOS}

Os dados apresentados na Tabela 1 mostram que a frequência de LP foi de 29\% (n=9) na clínica médica, 16\% $(n=4)$ na clínica cirúrgica e 53,8\% (n=7) na observação do pronto-socorro.

Tabela 1. Ocorrências de lesões por pressão (LP) durante a internação em um hospital-escola. Pouso Alegre, Minas Gerais, Brasil, 2016.

\begin{tabular}{cccc} 
LP & \multicolumn{3}{c}{ Setor } \\
\cline { 2 - 4 } & $\begin{array}{c}\text { Clínica médica } \\
\mathbf{n}(\%)\end{array}$ & $\begin{array}{c}\text { Clínica cirúrgica } \\
\mathbf{n}(\%)\end{array}$ & $\begin{array}{c}\text { Observação } \\
\mathbf{n}(\%)\end{array}$ \\
\hline Sim & $9(29,0)$ & $4(16,0)$ & $7(53,8)$ \\
\hline Não & $22(71,0)$ & $21(84,0)$ & $6(46,2)$ \\
\hline Total & $31(100,0)$ & $25(100,0)$ & $13(100,0)$ \\
\hline
\end{tabular}

$\mathrm{Na}$ Tabela 2, observamos que a maioria dos participantes do estudo que desenvolveram LP era da faixa etária entre 60 e 96 anos, do gênero feminino e da cor branca, e não houve diferença estatística entre as variáveis.

$\mathrm{Na}$ Tabela 3, podemos verificar a ocorrência de LP relacionadas aos dados clínicos em pacientes que participaram do estudo. Entre os pacientes internados na clínica médica, seis $(35,3 \%)$ tinham baixo peso e dois $(28,60 \%)$ sobrepeso. Entre os pacientes internados 
na clínica cirúrgica, três (25\%) tinham peso normal e um (20\%) obesidade. Já entre os pacientes internados na observação, quatro (80\%) estavam abaixo do peso. A maioria dos pacientes que participaram do estudo era diabética e não tinha hipertensão. Não houve diferença estatística entre as variáveis.

Tabela 2. Ocorrências de lesões por pressão (LP) durante a internação em um hospital-escola, segundo variáveis sociodemográficas. Pouso Alegre, Minas Gerais, Brasil, 2016.

\begin{tabular}{|c|c|c|c|c|c|c|c|}
\hline \multirow{2}{*}{\multicolumn{2}{|c|}{ Variáveis }} & \multicolumn{2}{|c|}{ Clinica médica } & \multicolumn{2}{|c|}{ Clínica cirúrgica } & \multicolumn{2}{|c|}{ Observação } \\
\hline & & Com LP & Sem LP & Com LP & Sem LP & Com LP & Sem LP \\
\hline & & \multicolumn{6}{|c|}{ n (\%) } \\
\hline \multirow{5}{*}{$\begin{array}{l}\text { Faixa etária } \\
\text { (anos) }\end{array}$} & 22 a 39 & $1(100,0)$ & $0(0,0)$ & $0(0,0)$ & $7(100,0)$ & $0(0,0)$ & $0(0,0)$ \\
\hline & 40 a 59 & $2(22,2)$ & $7(77,8)$ & $0(0,0)$ & $5(100,0)$ & $1(33,3)$ & $2(66,7)$ \\
\hline & 60 a 79 & $3(30,0)$ & $7(70,0)$ & $3(42,9)$ & $4(57,1)$ & $2(50,0)$ & $2(50,0)$ \\
\hline & 80 a 96 & $3(27,3)$ & $8(72,7)$ & $1(16,7)$ & $5(83,3)$ & $4(66,7)$ & $2(33,3)$ \\
\hline & $\mathrm{p}$ & \multicolumn{2}{|c|}{0,231} & \multicolumn{2}{|c|}{ 0,091 } & \multicolumn{2}{|c|}{0,301} \\
\hline \multirow{3}{*}{ Sexo } & Masculino & $3(30,0)$ & $7(70,0)$ & $2(16,7)$ & $10(83,3)$ & $3(60,0)$ & $2(40,0)$ \\
\hline & Feminino & $6(28,6)$ & $15(71,4)$ & $2(15,4)$ & $11(84,6)$ & $4(50,0)$ & $4(50,0)$ \\
\hline & $p$ & \multicolumn{2}{|c|}{0,549} & \multicolumn{2}{|c|}{0,761} & \multicolumn{2}{|c|}{0,781} \\
\hline \multirow{4}{*}{ Etnia } & Branca & $9(31,0)$ & $20(69,0)$ & $4(17,4)$ & $19(82,6)$ & $6(50,0)$ & $6(50,0)$ \\
\hline & Negra & $0(0,0)$ & $2(100,0)$ & $0(0,0)$ & $1(100,0)$ & $0(0,0)$ & $0(0,0)$ \\
\hline & Parda & $0(0,0)$ & $0(0,0)$ & $0(0,0)$ & $1(100,0)$ & $1(100,0)$ & $0(0,0)$ \\
\hline & $p$ & \multicolumn{2}{|c|}{0,531} & \multicolumn{2}{|c|}{0,912} & \multicolumn{2}{|c|}{0,901} \\
\hline
\end{tabular}

Nível de significância estatística $(p<0,05)$.

Tabela 3. Ocorrências de lesões por pressão (LP) durante a internação em um hospital-escola, segundo as variáveis clínicas. Pouso Alegre, Minas Gerais, Brasil, 2016.

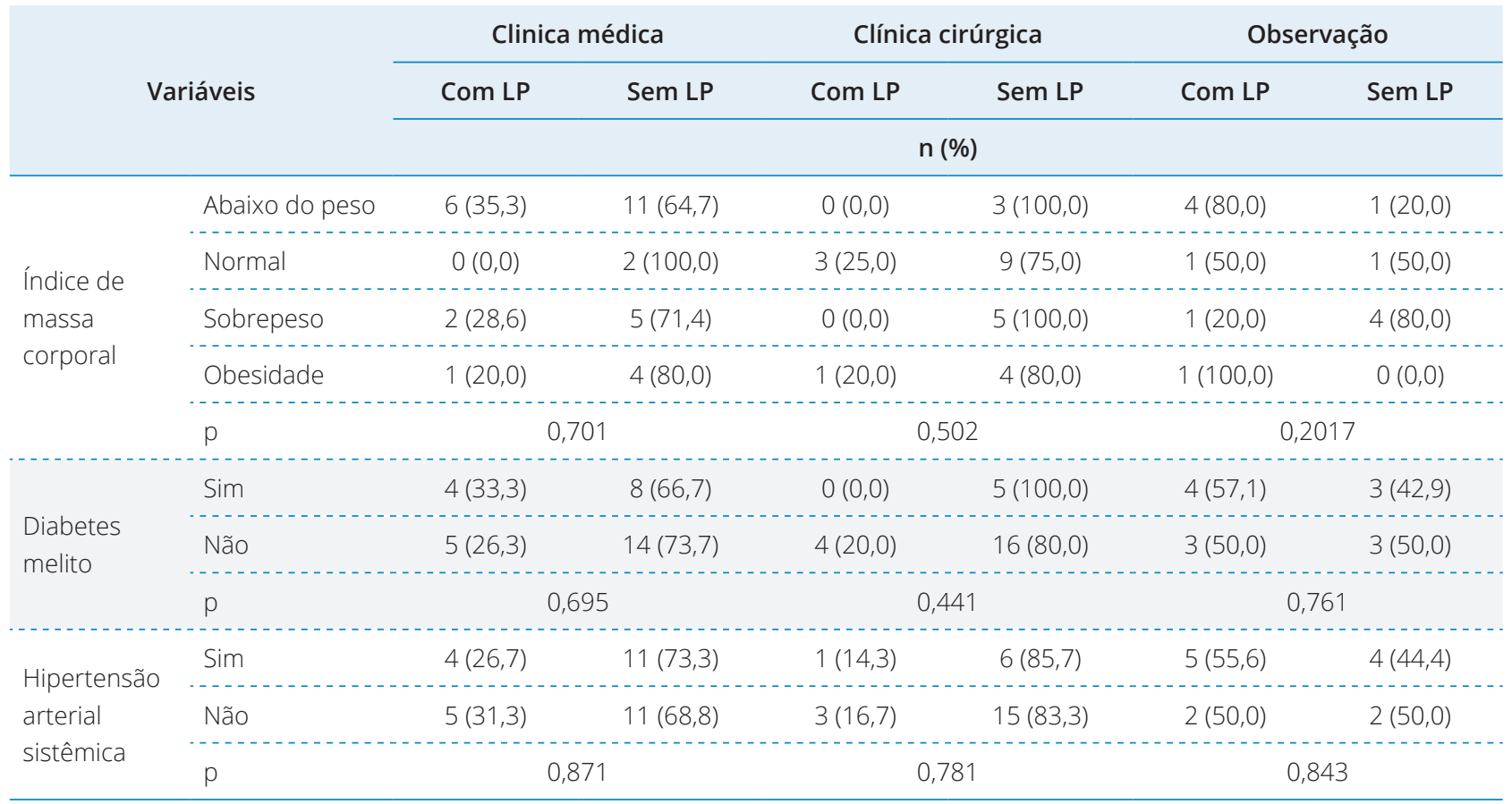

Nível de significância estatística $(p<0,05)$. 
A Tabela 4 mostra que todos os pacientes que participaram do estudo e que desenvolveram LP apresentaram risco moderado a elevado para o desenvolvimento dessa condição, segundo os escores da escala de Braden. Não houve diferença estatística entre as variáveis.
A Tabela 5 mostra os fatores de risco dos pacientes que participaram da pesquisa e que desenvolveram LP; todos estavam restritos ao leito, faziam uso de cateteres ou dispositivos e de droga vasoativa, usavam fralda, estavam em ventilação mecânica, sedados, em inconsciência e jejum alimentar e com período de internação acima de 10 dias.

Tabela 4. Ocorrências das lesões por pressão (LP) durante a internação em um hospital-escola, segundo a escala de Braden. Pouso Alegre, Minas Gerais, Brasil, 2016.

\begin{tabular}{|c|c|c|c|c|}
\hline \multirow{3}{*}{ Setor } & \multirow{3}{*}{ LP } & \multicolumn{3}{|c|}{ Escala de Braden } \\
\hline & & Moderado & Elevado & $p$ \\
\hline & & \multicolumn{3}{|c|}{ n (\%) } \\
\hline & $\operatorname{Sim}$ & $2(25,0)$ & $7(30,4)$ & \\
\hline \multirow[t]{3}{*}{ Clínica médica } & Não & $6(75,0)$ & $16(69,6)$ & 0,321 \\
\hline & Total & $8(100,0)$ & $23(100,0)$ & \\
\hline & $\mathrm{Sim}$ & $1(7,1)$ & $3(27,3)$ & \\
\hline \multirow[t]{3}{*}{ Clínica cirúrgica } & Não & $13(92,9)$ & $8(72,7)$ & 0,231 \\
\hline & Total & $14(100,0)$ & $11(100,0)$ & \\
\hline & $\mathrm{Sim}$ & $0(0,0)$ & $7(58,3)$ & \\
\hline \multirow[t]{2}{*}{ Observação } & Não & $1(100,0)$ & $5(41,7)$ & 0,431 \\
\hline & Total & $1(100,0)$ & $12(100,0)$ & \\
\hline
\end{tabular}

Teste exato de Fisher. Nível de significância estatística $(p<0,05)$.

Tabela 5. Ocorrências das lesões por pressão (LP) durante a internação em um hospital-escola, segundo os fatores de risco para LP. Pouso Alegre, Minas Gerais, Brasil, 2016.

\begin{tabular}{|c|c|c|c|c|c|c|c|}
\hline \multirow{3}{*}{ Variáveis } & & \multicolumn{2}{|c|}{ Clinica médica } & \multicolumn{2}{|c|}{ Clínica cirúrgica } & \multicolumn{2}{|c|}{ Observação } \\
\hline & & Com LP & Sem LP & Com LP & Sem LP & Com LP & Sem LP \\
\hline & & \multicolumn{6}{|c|}{ n (\%) } \\
\hline \multirow{3}{*}{ Restringido no leito } & $\operatorname{Sim}$ & $8(26,7)$ & $22(73,3)$ & $4(17,4)$ & $19(82,6)$ & $7(53,8)$ & $6(46,2)$ \\
\hline & Não & $1(100,0)$ & $0(0,0)$ & $0(0,0)$ & $2(100,0)$ & $0(0,0)$ & $0(0,0)$ \\
\hline & $\mathrm{p}$ & \multicolumn{2}{|c|}{0,201} & \multicolumn{2}{|c|}{ 0,301 } & \multicolumn{2}{|c|}{0,432} \\
\hline \multirow{4}{*}{$\begin{array}{l}\text { Uso de cateteres } \\
\text { ou dispositivo }\end{array}$} & $\operatorname{Sim}$ & $6(28,6)$ & $15(71,4)$ & $2(18,2)$ & $9(81,8)$ & $7(70,0)$ & $3(30,0)$ \\
\hline & Não & $3(30,0)$ & $7(70,0)$ & $2(14,3)$ & $12(85,7)$ & $0(0,0)$ & $3(100,0)$ \\
\hline & $\mathrm{p}$ & & & & & & \\
\hline & $\operatorname{Sim}$ & $9(29,0)$ & $22(71,0)$ & $3(20,0)$ & $12(80,0)$ & $6(53,8)$ & $6(46,2)$ \\
\hline \multirow[t]{3}{*}{ Uso de fralda } & Não & $0(0,0)$ & $0(0,0)$ & $1(10,0)$ & $9(90,0)$ & $0(0,0)$ & $0(0,0)$ \\
\hline & $\mathrm{p}$ & \multicolumn{2}{|c|}{0,321} & \multicolumn{2}{|c|}{0,379} & \multicolumn{2}{|c|}{0,401} \\
\hline & $\operatorname{Sim}$ & $2(50,0)$ & $2(50,0)$ & $0(0,0)$ & $0(0,0)$ & $2(50,0)$ & $2(50,0)$ \\
\hline \multirow[t]{3}{*}{ Jejum } & Não & $7(25,9)$ & $20(74,1)$ & $4(16,0)$ & $21(84,0)$ & $5(55,6)$ & $4(44,4)$ \\
\hline & $\mathrm{p}$ & \multicolumn{2}{|c|}{0,435} & \multicolumn{2}{|c|}{0,502} & \multicolumn{2}{|c|}{0,651} \\
\hline & Sim & $2(100,0)$ & $7(24,1)$ & $0(0,0)$ & $4(16,0)$ & $3(60,0)$ & $4(50,0)$ \\
\hline \multirow[t]{2}{*}{ Uso de sedação } & Não & $0(0,0)$ & $22(75,9)$ & $0(0,0)$ & $21(84,0)$ & $2(40,0)$ & $4(50,0)$ \\
\hline & $p$ & \multicolumn{2}{|c|}{$0,048 *$} & \multicolumn{2}{|c|}{0,901} & \multicolumn{2}{|c|}{0,903} \\
\hline
\end{tabular}


Tabela 5. Continuação...

\begin{tabular}{|c|c|c|c|c|c|c|c|}
\hline \multirow{3}{*}{$\begin{array}{l}\text { Uso de droga } \\
\text { vasoativa }\end{array}$} & Sim & $1(100,0)$ & $0(0,0)$ & $0(0,0)$ & $0(0,0)$ & $2(66,7)$ & $1(33,3)$ \\
\hline & Não & $8(26,7)$ & $22(73,3)$ & $4(16,0)$ & $21(84,0)$ & $5(50,0)$ & $5(50,0)$ \\
\hline & $p$ & \multicolumn{2}{|c|}{0,219} & \multicolumn{2}{|c|}{0,201} & \multicolumn{2}{|c|}{0,301} \\
\hline \multirow{3}{*}{ Agitação motora } & Sim & $1(20,0)$ & $4(80,0)$ & $0(0,0)$ & $0(0,0)$ & $3(100,0)$ & $0(0,0)$ \\
\hline & Não & $8(30,8)$ & $18(69,2)$ & $4(16,0)$ & $21(84,0)$ & $4(40,0)$ & $6(60,0)$ \\
\hline & $p$ & \multicolumn{2}{|c|}{0,158} & \multicolumn{2}{|c|}{0,192} & \multicolumn{2}{|c|}{0,184} \\
\hline \multirow{3}{*}{ Inconsciente } & $\operatorname{sim}$ & $3(75,0)$ & $1(25,0)$ & $1(50,0)$ & $1(50,0)$ & $3(60,0)$ & $2(40,0)$ \\
\hline & Não & $6(22,2)$ & $21(77,8)$ & $3(13,0)$ & $20(87,0)$ & $4(50,0)$ & $4(50,0)$ \\
\hline & $\mathrm{p}$ & \multicolumn{2}{|c|}{0,049} & \multicolumn{2}{|c|}{0,291} & \multicolumn{2}{|c|}{0,911} \\
\hline \multirow{4}{*}{$\begin{array}{l}\text { Período de } \\
\text { internação (dias) }\end{array}$} & $\begin{array}{l}\text { Menos de } \\
10\end{array}$ & $2(13,3)$ & $13(86,7)$ & $1(6,3)$ & $15(93,8)$ & $6(54,5)$ & $5(45,5)$ \\
\hline & $\begin{array}{l}10 \text { ou } \\
\text { mais }\end{array}$ & $7(43,8)$ & $9(56,3)$ & $3(33,3)$ & $6(66,7)$ & $1(50,0)$ & $1(50,0)$ \\
\hline & Total & $9(29,0)$ & $22(71,0)$ & $4(16,0)$ & $21(84,0)$ & $7(53,8)$ & $6(46,2)$ \\
\hline & p & \multicolumn{2}{|c|}{0,031 * } & \multicolumn{2}{|c|}{$0,039 *$} & \multicolumn{2}{|c|}{0,028} \\
\hline \multirow{3}{*}{$\begin{array}{l}\text { Uso de ventilação } \\
\text { mecânica }\end{array}$} & $\operatorname{sim}$ & $3(100,0)$ & $0(0,0)$ & $0(0,0)$ & $0(0,0)$ & $3(50,0)$ & $3(50,0)$ \\
\hline & Não & $6(21,4)$ & $22(78,6)$ & $4(16,0)$ & $21(84,0)$ & $4(57,1)$ & $3(42,9)$ \\
\hline & p & \multicolumn{2}{|c|}{$0,019 *$} & \multicolumn{2}{|c|}{0,901} & \multicolumn{2}{|c|}{0,991} \\
\hline
\end{tabular}

Teste exato de Fisher. * Significância estatística $(p<0,05)$.

\section{DISCUSSÃO}

$\mathrm{O}$ resultado deste estudo aponta uma frequência de ocorrência de LP de 29\% na clínica médica, $16 \%$ na clínica cirúrgica e 53,80\% na observação do pronto-socorro. Os resultados apresentam frequência elevada quando comparada aos de outros estudos ${ }^{18-20}$. Em estudo semelhante, os autores identificaram a frequência de LP de $22 \%$ em pacientes críticos $^{17}$. Em outro estudo, a frequência foi de $20,6 \%$ em setores de clínica cirúrgica ${ }^{18}$.

Para identificação da LP, todos os pacientes do estudo foram submetidos ao exame físico da pele e da escala de Braden, sendo que a maioria dos pacientes apresentou risco elevado para desenvolvimento de LP. Vários estudos corroboram os resultados obtidos ${ }^{11,16,19}$.

Os resultados de um estudo com objetivo de analisar os fatores de risco para o desenvolvimento de $\mathrm{LP}$ em pacientes adultos internados em centros de terapia intensiva, do qual fizeram parte 140 pacientes, mostraram, utilizando-se a escala de Braden, que pacientes internados por 15 dias ou mais apresentavam alguma categoria de risco. As maiores frequências de ocorrência de LP foram encontradas em pacientes que estavam nas categorias: percepção sensorial (completamente limitado), umidade (constantemente úmida), mobilidade (completamente imobilizado), atividade (acamado), nutrição (adequado) e fricção e cisalhamento (problema). Os autores concluíram que a utilização da escala de Braden é uma estratégia importante no cuidar de pacientes em terapia intensiva ${ }^{21}$.

Nesse estudo, a maioria dos pacientes apresentou os seguintes fatores de risco: idade acima de 61 anos, estar restringido no leito, uso de cateteres ou dispositivos, droga vasoativa, uso de fralda, estar em ventilação mecânica, sedados, inconscientes, em jejum alimentar, com período de internação acima de 10 dias e estar com baixo peso ou sobrepeso.

A diminuição do nível de consciência em decorrência de doenças de origem neurológica ou da percepção sensorial faz com que o cérebro apresente dificuldade em identificar o que está ocorrendo com o paciente por meio de estímulos nervosos, reduzindo sua percepção de desconforto e/ou dor, reduzindo, assim, a mobilidade e a atividade do paciente, o que pode ocasionar o desenvolvimento de LP. Dentre as modificações neurológicas e da mobilidade, destacam-se coma, imobilização e parestesias ${ }^{6}$. 
Em um estudo que avaliou a incidência de LP, os autores chegaram à conclusão de que um dos fatores que leva o paciente a desenvolver LP é sua limitação, já que $90 \%$ dos pacientes que desenvolveram essa condição apresentavam-se completamente limitados, enquanto, na mobilidade, 95\% dos sujeitos estavam imóveis ${ }^{19}$.

Em outro estudo, os autores concluíram que 95\% dos pacientes com nutrição inadequada apresentaram dificuldade em manter a integridade tissular da pele, além de problemas de regeneração e no processo cicatricial da pele ${ }^{23}$.

O conjunto de ações profiláticas tem início na identificação do paciente suscetível. O exame físico e a história clínica são, na maior parte dos casos, suficientes para a estimativa do risco, a qual determinará as intervenções a serem efetuadas. A estimativa do risco individual deve ser realizada periodicamente e o emprego de escalas de medida de risco pode ser útil no manejo preventivo ${ }^{24}$. Desse modo, ressalta-se que medidas profiláticas em relação às LP são de fundamental importância, principalmente para os pacientes criticamente enfermos. Para que a prevenção das LP seja efetivada, é necessário que haja treinamento adequado dos profissionais de saúde, juntamente com o apoio financeiro das instituições para disponibilizar treinamento e materiais adequados. Ainda com relação aos aspectos financeiros, cita-se o emprego de dispositivos redistribuidores da pressão que apresentam alto custo para as instituições; porém, a eficácia desses produtos faz com que esse investimento possa reduzir o tempo de internação e reflita positivamente na qualidade dos serviços de saúde prestados ${ }^{13-15}$.

\section{CONCLUSÃO}

Os resultados deste estudo mostram que a frequência de ocorrência de LP na clínica médica foi de 29\%, na clínica cirúrgica $16 \%$ e na observação do pronto-socorro $53,8 \%$. As LP desenvolveram-se predominantemente em pacientes do gênero feminino, raça branca e na faixa etária entre 60 e 96 anos. Os fatores de risco ou preditores do desenvolvimento de LP encontrados no estudo foram: período de internação igual ou superior a 10 dias, pacientes restringidos no leito, uso de cateteres ou dispositivos, uso de droga vasoativa, fraldas, ventilação mecânica, pacientes sedados, incontinência urinária e jejum alimentar. Com relação ao índice de massa corporal, a maioria dos pacientes apresentava baixo peso ou sobrepeso. A maioria dos pacientes apresentou risco elevado para desenvolvimento de LP, segundo os resultados da escala de Braden.

\section{CONTRIBUIÇÃO DOS AUTORES}

Conceitualização, Salomé GM; Metodologia, Salomé GM; Investigação, Barbosa JM e Salomé GM; Redação - Primeira versão, Barbosa JM e Salomé GM; Redação Revisão \& Edição, Salomé GM; Recursos, Salomé GM; Supervisão, Salomé GM.

\section{REFERÊNCIAS}

1. Edsberg LE, Black JM, Goldberg M, McNichol L, Moore L, Sieggreen M. Revised national pressure ulcer advisory panel pressure injury staging system: revised pressure injury staging system. J Wound Ostomy Continence Nurs. 2016;43(6):585-97. https://doi.org/10.1097/WON.000000000000028

2. Sieggree N. National pressure ulcer advisory panel responds to editorial. Adv Skin Wound Care. 2016; 29(12):535. https://doi. org/10.1097/01.ASW.0000508227.61503.f9

3. Dutra RA, Salomé GM, Alves JR, Perreira VO, Miranda FD, Valim FD, et al. Using transparent polyurethane film and hydrocolloid dressings to prevent pressure ulcers. J Wound Care. 2015; 24(6):268-75.https://doi.org/10.12968/ jowc.2015.24.6.268

4. Lourenco L, Blanes L, Salomé GM, Ferreira LM.
Quality of life and self-esteem in patients with paraplegia and pressure ulcers: a controlled crosssectional study. J Wound Care. 2014;23(6):331-7. https://doi.org/10.12968/jowc.2014.23.6.331

5. Dutra RA, Salomé GM, Leal LM, Alves MG, Moura JP, Silva AT, et al. Cost comparison of pressure ulcer preventive dressings: hydrocolloid dressing versus transparent polyurethane film. J Wound Care. 2016;25(11):635-40. https://doi.org/10.12968/ jowc.2016.25.11.635

6. Rogenski NM, Kurcgant P. Avaliação da concordância na aplicação da escala de Braden interobservadores. Acta Paul Enferm. 2012;25(1):24-8.

7. Schlüer $A B$. Pressure ulcers in maturing skin - A clinical perspective.J Tissue Viability. 2017;26(1):25.https://doi.org/10.1016/j.jtv.2016.10.001

8. de Lima EL, de Brito MJ, de Souza DM, Salomé 
GM, Ferreira LM. Cross-cultural adaptation and validation of the neonatal/infant Braden Q risk assessment scale. J Tissue Viability. 2016;25(1):5765. https://doi.org/10.1016/j.jtv.2015.12.004

9. de Araújo TM, de Araújo MF, Caetano JÁ. Using the Braden scale and photographs to assess pressure ulcer risk. Rev ESC Enferm USP. 2012:46(4):858-64.

10. Marques ADB, Branco JGO, Cavalcante RC, Brito MCC, Deus SRM, Luz MHBA. Conhecimento dos profissionais de saúde da família sobreúlcera por pressão. Rev Estima. 2017;15(2):63-73. https:// doi.org/10.5327/Z1806-3144201700020002

11. Queiroz AC, Mota DD, Bachion MM, Ferreira $A C$. Pressure ulcers in palliative home care patients: prevalence and characteristics. Rev Esc Enferm USP. 2014;48(2):264-71. https://doi. org/10.1590/S0080/6234201400002000010

12. Santos LRO, Avelino FVSD, Luz MHB, Cavalcante TB, Silva JLM, Santos CAPS. Demographic and clinical characteristics of intensive therapy units patients with pressure ulcer. I Nurs UFPE Online. 2016;10(Suppl. 1):225-31. https://doi.org/10.5205/reuol.7901-80479-1SP.1001sup201605

13. Vieira DS, Comassetto I, Faro ACM, Monteiro VGN, AlmeidaTG, Alves KMC. Interdisciplinary care to patients with pressure J Nurs UFPE Online. 2016;10(7):2428-35. https://doi.org/10.5205/ reuol.9106-80230-1-SM1007201617

14. Van Leen MW, Schols JM, Hovius SE, Halfens RJ. The effect of a simple 3-step pressure relieving strategy for preventing pressure ulcers: an explorative longitudinal study from 2002-2011. Wounds. 2014;26(10):285-92.

15. Van Leen M, Hovius S, Halfens R, Neyens J, Schols J. Pressure relief with visco-elastic foam or with combined static air overlay? A prospective, crossover randomized clinical trial in a Dutch nursing home. Wounds. 2013;25(10):287-92.

16. Paranhos WY, Santos VLCG. Avaliação do risco para úlceras de pressão por meio da escala de
Braden na língua portuguesa. Rev Esc Enferm USP. 1999;33(Espec):191-204.

17. Borghardt AT, Prado TN, Bicudo SDS, Castro DS, Bringuente ME. Úlcera por pressão em pacientes críticos: incidência e fatores associados. Rev Bras Enferm. 2016;69(3):460-7. https://doi. org/10.1590/0034-7167.2016690307i

18. Diniz EM, Morita ABPS, Paula MAB. Situação de risco para úlceras por pressão em uma unidade de assistência domiciliar. Rev Estima. 2016;14(2):53-60. https://doi.org/10.5327/Z18063144201600020002

19. Sousa Júnior BS, Silva CC, Duarte FHS, Mendonça AEO, Dantas DV. Análise das ações preventivas de úlceras por pressão por meio da escala de Braden. Rev Estima. 2017;15(1):10-8. https:// doi.org/10.5327/Z1806-3144201700010003

20. Ramos DO, Oliveira OS, Santos IV, Carvalho ES, Passos SS, Góis JA. Conhecimento de familiares acerca das úlceras por pressão e de seus direitos à reparação. Rev Baiana Enferm. 2014;28(1):23-30.

21. Almeida R, Giacomolli CMH, Coelho EL, Bittencourt VLL, Callegaro CC, Stumm EMF. High frequency generator in the treatment of injury by pressure in elderly. J Nurs UFPE Online. 2017;11(8):3136-42. https://.doi.org/10.5205/ reuol.11064-98681-4-ED.1108201719

22. Nogueira GA, Assad LG. Avaliação de risco para úlcera por pressão: contribuição para o cuidado de enfermagem na unidade de clínica médica. Rev Enferm UFPE. 2013;7(11):6462-70. https://doi.org/10.5205/reuol.3794-32322-1ED.0711201320

23. Mittag BF, Crozeta K, Roehrs H, Stocco J, Meier MJ. Continuing education about pressure ulcers. J Nurs UFPE Online. 2016;10(8):3020-3027. https://doi.org/10.5205/reuol.9373-82134-1RV1008201629

24. Luz SR, Lopacinski AC, Fraga R, Urban CA. Úlcera por pressão. Geriatr Gerontol. 2010;4(1):36-43. 